\title{
Bioastronomy (Commission 51)
}

\section{Introduction}

Commission 51 covers a very broad sweep of science, from astronomical topics, some of which overlap interests of some other commissions, to earth sciences, chemistry, biochemistry and even cognitive sciences concerned with the study of intelligence and animal behaviour. It is not feasible to attempt a complete coverage of all topics, even after due allowance for areas of overlap with other commissions. I have therefore been selective, attempting to include topics in which studies of particular interest have appeared during the triennium.

\section{R. D. Brown}

\section{Planetary discs: R. D. Brown}

Commission 51 has interest in the study of formation of new stars and planetary systems from the viewpoint of formation of potentially inhabitable locations, study of such systems also being of interest to other Commissions (e.g. 16) from other viewpoints. Commission 51 is also interested in the interstellar medium, molecular clouds and circumstellar envelopes from the aspect of origin of life and of habitable locations. The possibility that some aspects of interstellar chemistry might also be an indicator of the incipient formation of habitable locations (protoplanets) encourages me to report on some observations of celestial masers that could provide evidence of some embryo planetary systems.

Following a series of observations at Pushchino (Russia) of the $1.35 \mathrm{~cm} \mathrm{H} \mathrm{H}_{2} \mathrm{O}$ maser in $\mathrm{S} 140$ (Lekht et al. 1993), the maser emission being localised in three narrow, symmetrically spaced intervals of radial velocity, it was proposed that this constitutes maser amplification in a Keplerian disk surrounding a young star. It was estimated that the dimension of the masing part of the disk is $<30 \mathrm{AU}$, the mass of the central star being $<5 M_{\odot}$ and that the temporal variations of the spectral peaks' radial velocity could be caused by the clumps which could be either giant planets or protosatellites of giant protoplanets.

An interferometric study (Planesas et. al. 1992) of a hydrogen atom maser discovered (MartinPintado et al. 1989) in MWC3 shows that it probably is a disk seen edge on, whose masing part has a radius $\approx 40 \mathrm{AU}$. Monitoring of this multiline maser (Gordon 1992; Thum et al. 1993) indicates that the disk should be in Keplerian rotation and the thorough modelling of the observed spectral profile (Ponomarev et al. 1993) gave estimates of the density, temperature, turbulence and optical depth of the masing part of the disk. In this case the estimated mass of the central star is high - $26 M_{\odot}$ (Ponomarev et al. 1993).

In the last several years it has been possible to study methanol masers with the Australia Telescope, such interferometric observations giving better resolutions of groups of masers. The first publication (Norris et al. 1993a,b) reports ten alignments for which a plausible interpretation is that they are indications of such condensations and there are unpublished reports of further groups of aligned methanol masers. Typically the velocity gradients in these groupings are about $4 \mathrm{~km} \mathrm{~s}^{-1}$ over a linear extent of about $1000 \mathrm{AU}(5 \mathrm{mpc})$. To sustain such a gradient in Keplerian orbit would require a central mass of about $5 M_{\odot}$.

These alignments have been reported only for Class II methanol masers, which, as Cragg et al. (1992) have shown, need a pumping source of continuum radiation warmer than the gas kinetic temperature, and so are associated with a nearby infrared source which could be a protoplanetary condensation. If these interpretations stand up to further critical scrutiny then, when the maser excitation requirements are more quantitatively tied down by further modelling, we may have a particularly powerful probe of the physical conditions prevailing in such condensations. 


\section{References}

Cragg, D. M., Johns, K. P. Godfrey, P. D., Brown, R. D.: 1992, Ap. J., 259, 203-208.

Gordon, M. A. 1992, Ap. J. 387, 701.

Lekht, E. E., Likhachev, S. F., Sorochenko, R. 1., Strelnitski, V. S., 1993 Astron. Zhur., $70,731$.

Martin-Pintado, J., Thum, C. \& Bachiller, R.: 1989, A \& Ap., 222, L9-L11

Norris, R. P. Whiteoak, J. B. Caswell, J. L. Wieringa, M. H.: 1993a, Astrophysical Masers, A.W.

Clegg, G. E. Nedoluha (eds.) Springer-Verlag, Berlin Heidelberg, pp.203;

Norris, R. P. Whiteoak, J. B. Caswell, J. L. Wieringa, M. H. Gough, R. G. 1993b Ap. J., 412: 222.

Planesas, P., Martin-Pintado, J. \& Serabyn, E.: 1992, Ap. J., 386, L23.

Ponomarev, V. O., Smith, H. A. \& Strelnitski, V. S.: 1993, Ap. J., in press.

\section{Habitable zones and Planetary Habitability J. Heidmann}

The eventual success of life detection in the universe is strongly dependent on where we search for it. In particular for advanced cosmic life, the first lead we follow now is where Earth-like planets are more likely to exist. Early deliberations on habitability of planets were assembled by Dole (1970). Here we summarise recent developments in the study of this problem under three headings:

Stellar habitable zones: a suitable star: The range of "solar type" stars has been extended to later K and $M$ types (Doyle; Kasting et al., 1993) and also to double stars. Planets must be not too close and not too far from the parent, hence the concept of circumstellar habitable zones (Huang, 1959, 1960).

Planetary climatic role: Much new work has been done on the fundamental role of planetary atmospheres. Recent studies have been made on the green house effects (Kasting $e t$ al.) and extensive studies, theoretical and observational, on three nearby planetary bodies: Mars (Kasting, 1991; McKay, 1991; Jakosky et al. 1993; Bakeret al., 1991: Paige,, 1992), Venus (Komatsu et al., 1993; Robinson \& Wood, 1993) and Titan (McKay et al., 1993).

Environmental zones: Other habitability factors have been recognised. For instance, because of lethal effects from supernovae, only stars in the co-rotating region of spiral galaxies would be able to develop advanced forms of cosmic life. Their sky distribution is being evaluated as preferred search regions (Heidmann, 1993). Also, a fundamental role of our Moon in preventing large, chaotic variations of the inclination of the Earth axis, pernicious for life forms, has been recognised and subjected to detailed mathematical analysis (Laskar et al., 1993; Laskar \& Robutal, 1993). Eventual artificial activities such as terraforming Mars (McKay, et al., 1991) have also been investigated.

Recent reports : "Planetology \& the origins of life" and "Habitable zones" contain up-to-date information on habitable zones.

\section{References}

Baker, V. R., Strom, R. G., Gulick, V. C., Kargel, J. S., Komatsu, G., Kale, V. S., 1991, Nature, 353, 589.

Dole, S. H., 1970, "Habitable Planets for Man", 2nd ed. (Elsevier, New York).

Doyle, IAF 42nd Congress 92-615.

"Habitable zones", Ames, Res. Ctr., Jan 1994, ed. Doyle.

Heidmann, J., Acta Astronautica 1993 in press.

Jakosky, B. M., Henderson, B. G., Mellon, M. T., 1993, Icarus, 102, 286.

Kasting, J. F., 1991, Icarus , 94,1.

Kasting. J. F., Whitmire, D. P., Reynolds, R. T., 1993, Icarus, 101, 108.

Komatsu, G., Baker, V. R., Gulick, V. C., 1993, Icarus, 102,1. 


\section{References}

Cragg, D. M., Johns, K. P. Godfrey, P. D., Brown, R. D.: 1992, Ap. J., 259, 203-208.

Gordon, M. A. 1992, Ap. J. 387, 701.

Lekht, E. E., Likhachev, S. F., Sorochenko, R. 1., Strelnitski, V. S., 1993 Astron. Zhur., $70,731$.

Martin-Pintado, J., Thum, C. \& Bachiller, R.: 1989, A \& Ap., 222, L9-L11

Norris, R. P. Whiteoak, J. B. Caswell, J. L. Wieringa, M. H.: 1993a, Astrophysical Masers, A.W.

Clegg, G. E. Nedoluha (eds.) Springer-Verlag, Berlin Heidelberg, pp.203;

Norris, R. P. Whiteoak, J. B. Caswell, J. L. Wieringa, M. H. Gough, R. G. 1993b Ap. J., 412: 222.

Planesas, P., Martin-Pintado, J. \& Serabyn, E.: 1992, Ap. J., 386, L23.

Ponomarev, V. O., Smith, H. A. \& Strelnitski, V. S.: 1993, Ap. J., in press.

\section{Habitable zones and Planetary Habitability J. Heidmann}

The eventual success of life detection in the universe is strongly dependent on where we search for it. In particular for advanced cosmic life, the first lead we follow now is where Earth-like planets are more likely to exist. Early deliberations on habitability of planets were assembled by Dole (1970). Here we summarise recent developments in the study of this problem under three headings:

Stellar habitable zones: a suitable star: The range of "solar type" stars has been extended to later K and $M$ types (Doyle; Kasting et al., 1993) and also to double stars. Planets must be not too close and not too far from the parent, hence the concept of circumstellar habitable zones (Huang, 1959, 1960).

Planetary climatic role: Much new work has been done on the fundamental role of planetary atmospheres. Recent studies have been made on the green house effects (Kasting $e t$ al.) and extensive studies, theoretical and observational, on three nearby planetary bodies: Mars (Kasting, 1991; McKay, 1991; Jakosky et al. 1993; Bakeret al., 1991: Paige,, 1992), Venus (Komatsu et al., 1993; Robinson \& Wood, 1993) and Titan (McKay et al., 1993).

Environmental zones: Other habitability factors have been recognised. For instance, because of lethal effects from supernovae, only stars in the co-rotating region of spiral galaxies would be able to develop advanced forms of cosmic life. Their sky distribution is being evaluated as preferred search regions (Heidmann, 1993). Also, a fundamental role of our Moon in preventing large, chaotic variations of the inclination of the Earth axis, pernicious for life forms, has been recognised and subjected to detailed mathematical analysis (Laskar et al., 1993; Laskar \& Robutal, 1993). Eventual artificial activities such as terraforming Mars (McKay, et al., 1991) have also been investigated.

Recent reports : "Planetology \& the origins of life" and "Habitable zones" contain up-to-date information on habitable zones.

\section{References}

Baker, V. R., Strom, R. G., Gulick, V. C., Kargel, J. S., Komatsu, G., Kale, V. S., 1991, Nature, 353, 589.

Dole, S. H., 1970, "Habitable Planets for Man", 2nd ed. (Elsevier, New York).

Doyle, IAF 42nd Congress 92-615.

"Habitable zones", Ames, Res. Ctr., Jan 1994, ed. Doyle.

Heidmann, J., Acta Astronautica 1993 in press.

Jakosky, B. M., Henderson, B. G., Mellon, M. T., 1993, Icarus, 102, 286.

Kasting, J. F., 1991, Icarus , 94,1.

Kasting. J. F., Whitmire, D. P., Reynolds, R. T., 1993, Icarus, 101, 108.

Komatsu, G., Baker, V. R., Gulick, V. C., 1993, Icarus, 102,1. 
Laskar, J., Joutel, F., Robutel, P., 1993, Nature, 361, 615.

Laskar, J., Robutel, P., 1993, Nature, 361, 608.

McKay, C. P., 1991, Icarus, $91,93$.

McKay, C. P., Pollack, J. B., Lunine, J. I., Courtin, R., 1993, Icarus, 102, 88.

McKay, C. P., Toon, O. B., Kasting, J. F., 1991, Nature, 352, 489.

Paige, D. A., 1992, Nature, 356, 43.

"Planetology \& the origins of life", Cretell, Sept. 1993, ed. Raulin \& Bruston .

Robinson, C. A., Wood, J. A., 1993, Icarus, 102,26.

\section{Molecular clouds: W.M. Irvine}

The number of molecular species detected in the interstellar medium, not counting isotopic variants, has continued to grow over the past three years and now numbers nearly 100 . In the expanding envelopes around evolved stars, which return material to the interstellar medium, astronomers have identified the CP radical, the second phosphorus-containing astronomical molecule (Guélin et al. 1990); SiN, the first astronomical molecule with an $\mathrm{Si}-\mathrm{N}$ bond (Turner 1992); the first magnesium-containing interstellar molecule, MgNC (Kawaguchi et al. 1993); and the radical HCCN (Guélin and Cernicharo 1991). In interstellar molecular clouds there have been new identification or confirmations of previous tentative detections. These include the radical CCO (Ohishi et al. 1991, Brown et al. 1991), the $\mathrm{H}_{3} \mathrm{O}^{+}$ion, which is a crucial intermediary in the production of potentially major oxygen-bearing species $\mathrm{H}_{2} \mathrm{O}$ and $\mathrm{O}_{2}$ (Phillips et al. 1992); the ion $\mathrm{SO}^{+}$(Turner 1992, Irvine 1993), the radical $\mathrm{CH}_{2} \mathrm{~N}$ (Ohishi et al. 1993). Other newly identified species include isomers of previously known interstellar molecules, including HCCNC and CCCNH, both isomers of cyanoacetylene (Kawaguchi et al. 1992a, Kawaguchi et al. 1992b); and the two cumulenes $\mathrm{H}_{2} \mathrm{C}_{3}$ and $\mathrm{H}_{2} \mathrm{C}_{4}$, which are isomers of the pervasive interstellar molecule cyclic- $\mathrm{C}_{3} \mathrm{H}_{2}$ and of diacetylene, respectively (diacetylene itself has not been detected in the interstellar medium because of its lack of a permanent dipole moment; however, it may be presumed to be present based on the detection of the corresponding nitrile, $\mathrm{HC}_{5} \mathrm{~N}$ ).

Other previously know interstellar molecules have been found in new environments. Thus, both NO and NS have recently been detected for the first time in cold, dark clouds (McGonagle et al. 1990, McGonagle et al. 1993).

A potentially interesting probe of the pathways involved in molecular production is the orthopara ratio for species with two or more identical nuclei. Such ratios have now been reported for $\mathrm{H}_{2} \mathrm{CO}, \mathrm{H}_{2} \mathrm{CS}, \mathrm{C}_{3} \mathrm{H}_{2}, \mathrm{H}_{2} \mathrm{CCO}, \mathrm{H}_{2} \mathrm{C}_{3}, \mathrm{H}_{2} \mathrm{C}_{4}, \mathrm{CH}_{3} \mathrm{C}_{2} \mathrm{H}$, and $\mathrm{CH}_{3} \mathrm{CN}$. These results are summarised by Minh et al. (1993), who discusses the possible implications for exchange of material between icy grain mantles and the gas phase in interstellar clouds.

Models of interstellar chemistry, which previously have for the most part considered the timedependent chemical evolution of an interstellar cloud under conditions of constant physical properties, have now begun to address more systematically the coupled physics and chemistry evolving clouds and star-forming regions. Recent reviews in this area, which contain many references to recent work, include Harquist et al. (1993) and van Dischoeck et al. (1993).

\section{References}

Brown, R.D., Cragg, D.M., Godfrey, P.D., Irvine, W.M., McGonagle, D., and Ohishi, M. (1991), Origins of Life and Evolution of the Biosphere, 21, 399.

Cernicharo, J., Gottlieb, C.A, Guélin, M., Killian, T.C., Paubert, G., Thaddeus, P., and Vrtilek, J.M. (1991), Astrophys. J. (Letters) 368, L39.

Cernicharo, J., Gottlieb, C.A., Guélin, M., Killian, T.C., Thaddeus, P., and Vrtilek, J.M. (1991), Astrophys. J. (Letters) 368, L43.

Guélin, M., Cernicharo, J., Paubert, G., and Turner, B.E. (1990), Astron. Astrophys. 230, L9.

Guélin, M., and Cernicharo, J. (1991), Astron. Astrophys. 244, L21.

Hartquist, T.W. et al. (1993), Q.J.R.A.S. 34, 213.

Irvine, W.M. (1993), in Advances in Space Research, in press. 
Laskar, J., Joutel, F., Robutel, P., 1993, Nature, 361, 615.

Laskar, J., Robutel, P., 1993, Nature, 361, 608.

McKay, C. P., 1991, Icarus, $91,93$.

McKay, C. P., Pollack, J. B., Lunine, J. I., Courtin, R., 1993, Icarus, 102, 88.

McKay, C. P., Toon, O. B., Kasting, J. F., 1991, Nature, 352, 489.

Paige, D. A., 1992, Nature, 356, 43.

"Planetology \& the origins of life", Cretell, Sept. 1993, ed. Raulin \& Bruston .

Robinson, C. A., Wood, J. A., 1993, Icarus, 102,26.

\section{Molecular clouds: W.M. Irvine}

The number of molecular species detected in the interstellar medium, not counting isotopic variants, has continued to grow over the past three years and now numbers nearly 100 . In the expanding envelopes around evolved stars, which return material to the interstellar medium, astronomers have identified the CP radical, the second phosphorus-containing astronomical molecule (Guélin et al. 1990); SiN, the first astronomical molecule with an $\mathrm{Si}-\mathrm{N}$ bond (Turner 1992); the first magnesium-containing interstellar molecule, MgNC (Kawaguchi et al. 1993); and the radical HCCN (Guélin and Cernicharo 1991). In interstellar molecular clouds there have been new identification or confirmations of previous tentative detections. These include the radical CCO (Ohishi et al. 1991, Brown et al. 1991), the $\mathrm{H}_{3} \mathrm{O}^{+}$ion, which is a crucial intermediary in the production of potentially major oxygen-bearing species $\mathrm{H}_{2} \mathrm{O}$ and $\mathrm{O}_{2}$ (Phillips et al. 1992); the ion $\mathrm{SO}^{+}$(Turner 1992, Irvine 1993), the radical $\mathrm{CH}_{2} \mathrm{~N}$ (Ohishi et al. 1993). Other newly identified species include isomers of previously known interstellar molecules, including HCCNC and CCCNH, both isomers of cyanoacetylene (Kawaguchi et al. 1992a, Kawaguchi et al. 1992b); and the two cumulenes $\mathrm{H}_{2} \mathrm{C}_{3}$ and $\mathrm{H}_{2} \mathrm{C}_{4}$, which are isomers of the pervasive interstellar molecule cyclic- $\mathrm{C}_{3} \mathrm{H}_{2}$ and of diacetylene, respectively (diacetylene itself has not been detected in the interstellar medium because of its lack of a permanent dipole moment; however, it may be presumed to be present based on the detection of the corresponding nitrile, $\mathrm{HC}_{5} \mathrm{~N}$ ).

Other previously know interstellar molecules have been found in new environments. Thus, both NO and NS have recently been detected for the first time in cold, dark clouds (McGonagle et al. 1990, McGonagle et al. 1993).

A potentially interesting probe of the pathways involved in molecular production is the orthopara ratio for species with two or more identical nuclei. Such ratios have now been reported for $\mathrm{H}_{2} \mathrm{CO}, \mathrm{H}_{2} \mathrm{CS}, \mathrm{C}_{3} \mathrm{H}_{2}, \mathrm{H}_{2} \mathrm{CCO}, \mathrm{H}_{2} \mathrm{C}_{3}, \mathrm{H}_{2} \mathrm{C}_{4}, \mathrm{CH}_{3} \mathrm{C}_{2} \mathrm{H}$, and $\mathrm{CH}_{3} \mathrm{CN}$. These results are summarised by Minh et al. (1993), who discusses the possible implications for exchange of material between icy grain mantles and the gas phase in interstellar clouds.

Models of interstellar chemistry, which previously have for the most part considered the timedependent chemical evolution of an interstellar cloud under conditions of constant physical properties, have now begun to address more systematically the coupled physics and chemistry evolving clouds and star-forming regions. Recent reviews in this area, which contain many references to recent work, include Harquist et al. (1993) and van Dischoeck et al. (1993).

\section{References}

Brown, R.D., Cragg, D.M., Godfrey, P.D., Irvine, W.M., McGonagle, D., and Ohishi, M. (1991), Origins of Life and Evolution of the Biosphere, 21, 399.

Cernicharo, J., Gottlieb, C.A, Guélin, M., Killian, T.C., Paubert, G., Thaddeus, P., and Vrtilek, J.M. (1991), Astrophys. J. (Letters) 368, L39.

Cernicharo, J., Gottlieb, C.A., Guélin, M., Killian, T.C., Thaddeus, P., and Vrtilek, J.M. (1991), Astrophys. J. (Letters) 368, L43.

Guélin, M., Cernicharo, J., Paubert, G., and Turner, B.E. (1990), Astron. Astrophys. 230, L9.

Guélin, M., and Cernicharo, J. (1991), Astron. Astrophys. 244, L21.

Hartquist, T.W. et al. (1993), Q.J.R.A.S. 34, 213.

Irvine, W.M. (1993), in Advances in Space Research, in press. 
Kawaguchi, K., Kaifu, N., Ohishi, M., Ishikawa, S-I., Hirahara, Y., Yamamoto, S., Saito, S., Takano, S., Murakami, A., Vrtilek, J.M. Gottlieb, C.A. Thaddeus, P., and Irvine, W.M. (1991), Publ. Astron. Soc. Japan 43, 607.

Kawaguchi, K., Ohishi, M., Ishikawa, S-I., and Kaifu, N. (1992a), Astrophys. J. (Letters) 386, L51.

Kawaguchi, K., Takano, S., Ohishi, M., Ishikawa, S-I., Miyazawa, K., Kaifu, N., Yamashita, K., Yamamoto, S., Saito, S., Oshima, Y., and Endo, Y. (1992b), Astrophys. J. (Letters) 396, L49.

Kawaguchi, K., Kagi, E., Hirano, T., Takano, S., and Saito, S. (1993), Astrophys. J. (Letters) 406, L39.

McGonagle, D., Ziurys, L.M., Irvine, W.M. and Minh, Y.C. (1990), Astrophys. J. 359, 121.

McGonagle, D., Irvine, W.M., and Ohishi, M. (1993), in preparation.

Minh, Y.C., Irvine, W.M., Ohishi, M., Ishikawa, S., Saito, S., and Kaifu, N. (1993), Astron. Astrophys. 267, 229.

Ohishi, M., Suzuki, H., Ishikawa, S-I., Yamada, C., Kanamori, H., Irvine, W.M. Brown, R.D., Godfrey, P.D., and Kaifu, N. (1991), Astrophys. J. (Letters) 380, L39.

Ohishi, M., Irvine, W.M., McGonagle, D., Saito, S., and Yamamoto, S. (1993), in preparation.

Phillips, T.G., van Dishoeck, E., and Keene, J. (1992), Astrophys. J. 399, 533.

Turner, B.E. (1992), Astrophys. J. (Letters) 388, L35.

Turner, B.E. (1992), Astrophys. J. (Letters) 396, L107.

van Dishoeck, E.F. et al. (1993), in Protostars and Planets III, ed. E.H. Levy, J.I. Lunine, and M.S. Matthews, University of Arizona Press, Tucson, pp. 165-191.

\section{Chemical evolution of Earth: R. D. Brown}

Three distinct scenarios have been proposed for the prebiotic chemical evolution of the Earth's biosphere: the Miller-Urey hypothesis of the generation of prebiotic molecules by lightning discharges in the reduced atmosphere $\left(\mathrm{CH}_{4}, \mathrm{NH}_{3}, \mathrm{H}_{2} \mathrm{O}\right.$ and $\left.\mathrm{H}_{2}\right)$ of the young Earth; the cometary seeding hypothesis of Oro (Oro 1961, 1965; Brown 1977); the clay-based life and genetic takeover hypothesis of Cairns-Smith. The first of these has faced increasing difficulties as evidence has accumulated that the original atmosphere of the Earth was in an oxidised, rather than reduced, state.

Recent developments relating to the last of the three scenarios are summarised below. Here is outlined a recent proposal that increases the plausibility of the second of the scenarios and simultaneously turns a spotlight on giant planets as indicators of habitable zones in planetary systems.

When Oro made his suggestion it was generally felt that the amount of organic material arriving on the Earth's surface from cometary encounters would be relatively insignificant and swamped in the primeval seas. However Delsemme (1992) has now built a scenario, based on Safronov's (1969, 1972) demonstration that the giant planets, particularly Jupiter, were responsible for scattering asteroids. This prevented formation of another large planet and led to the accretion by the terrestrial planets of volatile planetesimal material that is more than sufficient to account for the mass of the present biosphere. Delsemme estimates that such exogenous origins of water would provide a liquid layer $15 \mathrm{~km}$ thick on the Earth's surface, amounting to $7.5 \times 10^{21} \mathrm{~kg}$, along with $1 \times 10^{21} \mathrm{~kg}$ of carbon and a similar mass of gaseous atmosphere.

Delsemme summarises evidence, too extensive to reproduce here, that the Earth was formed by hot accretion and that any early atmosphere of original volatiles would have been evaporated and dispersed. The subsequent accretion of carbonaceous material by the Earth would have included prebiotic molecules such as aminoacids, purines and pyrimidines, probably (Anders 1971) generated by the Fischer Tropsch process in a gas of solar composition. The complexity of this chemistry, particularly as it relates to chondrites, has been discussed critically by Prin and Fegley (1989) and by Cronin et. al. (1988).

Anders, E.: 1971, Ann. Rev. Astron. Astrophys. 9, 1-34.

Brown, R. D.: 1977, Interdisciplin. Sci. Rev., 2, 124-139. 
Kawaguchi, K., Kaifu, N., Ohishi, M., Ishikawa, S-I., Hirahara, Y., Yamamoto, S., Saito, S., Takano, S., Murakami, A., Vrtilek, J.M. Gottlieb, C.A. Thaddeus, P., and Irvine, W.M. (1991), Publ. Astron. Soc. Japan 43, 607.

Kawaguchi, K., Ohishi, M., Ishikawa, S-I., and Kaifu, N. (1992a), Astrophys. J. (Letters) 386, L51.

Kawaguchi, K., Takano, S., Ohishi, M., Ishikawa, S-I., Miyazawa, K., Kaifu, N., Yamashita, K., Yamamoto, S., Saito, S., Oshima, Y., and Endo, Y. (1992b), Astrophys. J. (Letters) 396, L49.

Kawaguchi, K., Kagi, E., Hirano, T., Takano, S., and Saito, S. (1993), Astrophys. J. (Letters) 406, L39.

McGonagle, D., Ziurys, L.M., Irvine, W.M. and Minh, Y.C. (1990), Astrophys. J. 359, 121.

McGonagle, D., Irvine, W.M., and Ohishi, M. (1993), in preparation.

Minh, Y.C., Irvine, W.M., Ohishi, M., Ishikawa, S., Saito, S., and Kaifu, N. (1993), Astron. Astrophys. 267, 229.

Ohishi, M., Suzuki, H., Ishikawa, S-I., Yamada, C., Kanamori, H., Irvine, W.M. Brown, R.D., Godfrey, P.D., and Kaifu, N. (1991), Astrophys. J. (Letters) 380, L39.

Ohishi, M., Irvine, W.M., McGonagle, D., Saito, S., and Yamamoto, S. (1993), in preparation.

Phillips, T.G., van Dishoeck, E., and Keene, J. (1992), Astrophys. J. 399, 533.

Turner, B.E. (1992), Astrophys. J. (Letters) 388, L35.

Turner, B.E. (1992), Astrophys. J. (Letters) 396, L107.

van Dishoeck, E.F. et al. (1993), in Protostars and Planets III, ed. E.H. Levy, J.I. Lunine, and M.S. Matthews, University of Arizona Press, Tucson, pp. 165-191.

\section{Chemical evolution of Earth: R. D. Brown}

Three distinct scenarios have been proposed for the prebiotic chemical evolution of the Earth's biosphere: the Miller-Urey hypothesis of the generation of prebiotic molecules by lightning discharges in the reduced atmosphere $\left(\mathrm{CH}_{4}, \mathrm{NH}_{3}, \mathrm{H}_{2} \mathrm{O}\right.$ and $\left.\mathrm{H}_{2}\right)$ of the young Earth; the cometary seeding hypothesis of Oro (Oro 1961, 1965; Brown 1977); the clay-based life and genetic takeover hypothesis of Cairns-Smith. The first of these has faced increasing difficulties as evidence has accumulated that the original atmosphere of the Earth was in an oxidised, rather than reduced, state.

Recent developments relating to the last of the three scenarios are summarised below. Here is outlined a recent proposal that increases the plausibility of the second of the scenarios and simultaneously turns a spotlight on giant planets as indicators of habitable zones in planetary systems.

When Oro made his suggestion it was generally felt that the amount of organic material arriving on the Earth's surface from cometary encounters would be relatively insignificant and swamped in the primeval seas. However Delsemme (1992) has now built a scenario, based on Safronov's (1969, 1972) demonstration that the giant planets, particularly Jupiter, were responsible for scattering asteroids. This prevented formation of another large planet and led to the accretion by the terrestrial planets of volatile planetesimal material that is more than sufficient to account for the mass of the present biosphere. Delsemme estimates that such exogenous origins of water would provide a liquid layer $15 \mathrm{~km}$ thick on the Earth's surface, amounting to $7.5 \times 10^{21} \mathrm{~kg}$, along with $1 \times 10^{21} \mathrm{~kg}$ of carbon and a similar mass of gaseous atmosphere.

Delsemme summarises evidence, too extensive to reproduce here, that the Earth was formed by hot accretion and that any early atmosphere of original volatiles would have been evaporated and dispersed. The subsequent accretion of carbonaceous material by the Earth would have included prebiotic molecules such as aminoacids, purines and pyrimidines, probably (Anders 1971) generated by the Fischer Tropsch process in a gas of solar composition. The complexity of this chemistry, particularly as it relates to chondrites, has been discussed critically by Prin and Fegley (1989) and by Cronin et. al. (1988).

Anders, E.: 1971, Ann. Rev. Astron. Astrophys. 9, 1-34.

Brown, R. D.: 1977, Interdisciplin. Sci. Rev., 2, 124-139. 
Cronin, J. R., Pizarello, S., and Cruikshank, D. P.: 1988 'Meteorites and the Early Solar System',Kerrige and Matthews (eds.) Univ. of Arizona Press, Tucson, pp.819-857.

Delsemme, A. H.: 1992, Orig. Life and Evol. Bio., 21, 279-298.

Oro, J. 1961 Nature, 190, 389-390.

Oro, J. 1965 "The Origins of Prebiological Systems and their Molecular Matrices', S. W. Fox 9ed.) Academic Press, New York, pp. 137-162.

Prin, R. G. and Fegley, B.: 1989 'Origin of Planet and Satellite Atmospheres',136, S.K. Atreya et. al. (eds.) Univ. of Arizona Press, Tucson, pp.78-136.

Safronov, V. S. 1969 'Evolution of Protoplanetary Cloud and Formation of the Earth and the Planets', translated in NASA TTF-667-1972.

\section{Developments in Chemical Origins of Life 1990-1993 A. G. Cairns-Smith}

An enthusiasm of the late 80s for the idea that life on the Earth could have started with nothing but RNA molecules (acting as catalysts for their own replication) has been tempered by an appreciation of the difficulties of making the activated nucleotide necessary to set up such an "RNA world". Attention is now being paid to the idea that evolution might have got going with a somewhat different chemistry.

RNA-type molecules with modified backbone have been considered as primordial genetic material (reviewed by Schwartz 1992). Such research is of interest in its own right in helping to define the range of possible replicating molecules (reviewed extensively by Orgel, 1992). There have indeed been moves sideways, as it were, towards the consideration of models which might allow evolutionary processes to be set going in the laboratory based on molecules which may have little if any connection with our biochemistry. Both Rebek and von Kiedrowski and their colleagues have made different kinds of artificial replicating molecule (Rotello, Hong \& Rebek, 1991; Terfort \& von Kiedrowski, 1992) - a considerable landmark even if as yet their molecules are too simple to have any evolutionary potential.

Off the tack of primary genetic materials De Duve (1991) has made a case for sulphur, e.g. in thioesters, as well as iron, as having had more dominant biochemical roles to begin with. This fits with Wächtershäuser's (1990) model of a pre-evolution on the surfaces of iron sulphide minerals.

One place where such minerals are to be found are hydrothermal systems where hot solutions circulate within the Earth's crust, and the idea that life might have started in some such relatively protected environment has been re-considered in some detail (Holm, 1992).

The possible roles of comets in the origin of life (as providers and/or destroyers) have been similarly re-considered (Thomas, 1991/2). Perhaps the most interesting suggestion here is that comets may have been responsible not for providing biochemicals as such but, much more fundamentally, for giving the Earth its supplies of carbon, nitrogen and water, see Section 4.

\section{References}

de Duve, C. (1991). Blueprint for a Cell. Burlington, North Caroline: Neil Patterson.

Holm, N.G. (editor) 1992. Marine hydrothermal systems and the origin of life. Special issue of Origins of Life and Evolution of the Biosphere, 22, 1, 242.

Orgel, I. E. (1992). Molecular replication. Nature, 358, 203-209.

Rotello, V., Hong, J-I. \& Rebek Jr, J. (1991). J. Am. Chem. Soc. 113, 9422-9423.

Schwartz, A.W. (1992). Getting the RNA world started. In Frontiers of Life ed. J \& K Tian Thanh Van, Cif-sur-Yvette: Editions Frontiers, pp 145-156.

Terfurt, A. \& von Kiedrowski, C. 1992. Self-replication by condensation of 3-aminobenzamidines and 2-formylphenoxyacetic acids. Angew. Chem. int. Ed. Engl. 31, 554-655.

Thomas, P.J. (editor) 1991/2. Comets and the origin and evolution of life. Special issue of Origins of Life and Evolution of the Biosphere, 21, 265-436. 
Cronin, J. R., Pizarello, S., and Cruikshank, D. P.: 1988 'Meteorites and the Early Solar System',Kerrige and Matthews (eds.) Univ. of Arizona Press, Tucson, pp.819-857.

Delsemme, A. H.: 1992, Orig. Life and Evol. Bio., 21, 279-298.

Oro, J. 1961 Nature, 190, 389-390.

Oro, J. 1965 "The Origins of Prebiological Systems and their Molecular Matrices', S. W. Fox 9ed.) Academic Press, New York, pp. 137-162.

Prin, R. G. and Fegley, B.: 1989 'Origin of Planet and Satellite Atmospheres',136, S.K. Atreya et. al. (eds.) Univ. of Arizona Press, Tucson, pp.78-136.

Safronov, V. S. 1969 'Evolution of Protoplanetary Cloud and Formation of the Earth and the Planets', translated in NASA TTF-667-1972.

\section{Developments in Chemical Origins of Life 1990-1993 A. G. Cairns-Smith}

An enthusiasm of the late 80s for the idea that life on the Earth could have started with nothing but RNA molecules (acting as catalysts for their own replication) has been tempered by an appreciation of the difficulties of making the activated nucleotide necessary to set up such an "RNA world". Attention is now being paid to the idea that evolution might have got going with a somewhat different chemistry.

RNA-type molecules with modified backbone have been considered as primordial genetic material (reviewed by Schwartz 1992). Such research is of interest in its own right in helping to define the range of possible replicating molecules (reviewed extensively by Orgel, 1992). There have indeed been moves sideways, as it were, towards the consideration of models which might allow evolutionary processes to be set going in the laboratory based on molecules which may have little if any connection with our biochemistry. Both Rebek and von Kiedrowski and their colleagues have made different kinds of artificial replicating molecule (Rotello, Hong \& Rebek, 1991; Terfort \& von Kiedrowski, 1992) - a considerable landmark even if as yet their molecules are too simple to have any evolutionary potential.

Off the tack of primary genetic materials De Duve (1991) has made a case for sulphur, e.g. in thioesters, as well as iron, as having had more dominant biochemical roles to begin with. This fits with Wächtershäuser's (1990) model of a pre-evolution on the surfaces of iron sulphide minerals.

One place where such minerals are to be found are hydrothermal systems where hot solutions circulate within the Earth's crust, and the idea that life might have started in some such relatively protected environment has been re-considered in some detail (Holm, 1992).

The possible roles of comets in the origin of life (as providers and/or destroyers) have been similarly re-considered (Thomas, 1991/2). Perhaps the most interesting suggestion here is that comets may have been responsible not for providing biochemicals as such but, much more fundamentally, for giving the Earth its supplies of carbon, nitrogen and water, see Section 4.

\section{References}

de Duve, C. (1991). Blueprint for a Cell. Burlington, North Caroline: Neil Patterson.

Holm, N.G. (editor) 1992. Marine hydrothermal systems and the origin of life. Special issue of Origins of Life and Evolution of the Biosphere, 22, 1, 242.

Orgel, I. E. (1992). Molecular replication. Nature, 358, 203-209.

Rotello, V., Hong, J-I. \& Rebek Jr, J. (1991). J. Am. Chem. Soc. 113, 9422-9423.

Schwartz, A.W. (1992). Getting the RNA world started. In Frontiers of Life ed. J \& K Tian Thanh Van, Cif-sur-Yvette: Editions Frontiers, pp 145-156.

Terfurt, A. \& von Kiedrowski, C. 1992. Self-replication by condensation of 3-aminobenzamidines and 2-formylphenoxyacetic acids. Angew. Chem. int. Ed. Engl. 31, 554-655.

Thomas, P.J. (editor) 1991/2. Comets and the origin and evolution of life. Special issue of Origins of Life and Evolution of the Biosphere, 21, 265-436. 
Wächtershäuser, G. (1990). The case for the chemotropic origin of life in an iron-sulphur world. Origins of Life and Evolution of the Biosphere, 20, 173-176.

\section{Intelligence, Cognition and Communication in Other Species: Diana Reiss}

As we search for evidence of intelligent life elsewhere in the universe we face the question of whether we could recognise and evaluate intelligence in extraterrestrials. Reiss (198) and Marino (1993) have suggested that bioastronomers, biologists and psychologists share common problems in exploring non-human intelligence and communication in extraterrestrials or other non-human terrestrial species. Initially we face decoding issues which include 1) signal detection, 2) signal structural analysis, and 3) signal function. (Reiss, 1993a). Secondly, in investigating intelligence and evolutionary continuity of mental experience in other species we are challenged in finding appropriate measures and frameworks for assessing information processing strategies used by other species with body forms and environmental histories different from our own. Past views that humans are unique as a symbol and tool using species are currently being challenged as we have reports of highly divergent species such as chimpanzees (Gardner \& Gardner, 1969; Fouts, 1973, Savage-Rumbaugh, 1986), African grey parrots (Pepperberg, 1981), California sea lions (Schusterman \& Krieger, 1984), and bottlenose dolphins (Reiss \& McCowan), 1993; Richards, Woltz \& Herman, 1984) comprehending and/or producing symbolic codes. Several of these species have also demonstrated varying degrees of tool use. There is increasing evidence of tool use by nonhuman species (Seyfarth, Cheney, and Marier, 1980; Owings and Leger, 1980).

Evidence for vocal learning has been found in three highly divergent species, humans, birds (Marler, 1969) and dolphins (Reiss and McCowan, 1993). Research in this area strongly suggests that common strategies may be used in acquiring vocal repertoires across species.

Studies have demonstrated that chimpanzees, and orangutans as well as humans can recognise their reflections in mirrors (for review see Povinelli, 1987). Marino, Reiss and Gallup (1993) have found suggestive evidence of self-recognition in bottlenose dolphins. Diverse species such as elephants (Povinelli, 1989), and African grey parrots (Pepperberg, 1993) have demonstrated mirror guided behaviour in accomplishing tasks.

Evidence for conceptual quantitative abilities has been reported in the African grey parrot (Pepperberg, 1987) and the chimpanzee (Boysen and Berntson, 1989).

There is clearly much more complexity and plasticity found in the communication systems of other species that previously thought. More research is needed with more diverse species to more adequately access the extent to which referential communication, quantitative abilities and complex information processing has developed throughout the biological world.

\section{References}

Boysen, S.T. and Berntson, G,G. (1989). Numerical Competence in a chimpanzee (Pan troglodytes). Journal of Comparative Psychology, 103, 23-30.

Fouts, R.S. (1973). Acquisition and testing of gestural signs in four young chimpanzees. Science, 180: 978-980.

Gardner, R.A. and Gardner, B.T. (1969). Teaching sign language to a chimpanzee. Science, 1965: 664-672.

Marion, L.A., Reiss, D. and Gallup, Jr. G.G. (1993). A test of self recognition in dolphins: implications for comparative investigations of highly dissimilar species. In: Selfawareness in Humans and Nonhumans. S. Parker, R. Mitchell, and M. Boccia (eds.) Cambridge University Press.

Marino, L.A., (1993) SETI begins at home: Can we define and measure intelligence among species $\mathrm{n}$ this planet? 1993 Bioastronomy Symposium, University of California, Santa Cruz. 
Wächtershäuser, G. (1990). The case for the chemotropic origin of life in an iron-sulphur world. Origins of Life and Evolution of the Biosphere, 20, 173-176.

\section{Intelligence, Cognition and Communication in Other Species: Diana Reiss}

As we search for evidence of intelligent life elsewhere in the universe we face the question of whether we could recognise and evaluate intelligence in extraterrestrials. Reiss (198) and Marino (1993) have suggested that bioastronomers, biologists and psychologists share common problems in exploring non-human intelligence and communication in extraterrestrials or other non-human terrestrial species. Initially we face decoding issues which include 1) signal detection, 2) signal structural analysis, and 3) signal function. (Reiss, 1993a). Secondly, in investigating intelligence and evolutionary continuity of mental experience in other species we are challenged in finding appropriate measures and frameworks for assessing information processing strategies used by other species with body forms and environmental histories different from our own. Past views that humans are unique as a symbol and tool using species are currently being challenged as we have reports of highly divergent species such as chimpanzees (Gardner \& Gardner, 1969; Fouts, 1973, Savage-Rumbaugh, 1986), African grey parrots (Pepperberg, 1981), California sea lions (Schusterman \& Krieger, 1984), and bottlenose dolphins (Reiss \& McCowan), 1993; Richards, Woltz \& Herman, 1984) comprehending and/or producing symbolic codes. Several of these species have also demonstrated varying degrees of tool use. There is increasing evidence of tool use by nonhuman species (Seyfarth, Cheney, and Marier, 1980; Owings and Leger, 1980).

Evidence for vocal learning has been found in three highly divergent species, humans, birds (Marler, 1969) and dolphins (Reiss and McCowan, 1993). Research in this area strongly suggests that common strategies may be used in acquiring vocal repertoires across species.

Studies have demonstrated that chimpanzees, and orangutans as well as humans can recognise their reflections in mirrors (for review see Povinelli, 1987). Marino, Reiss and Gallup (1993) have found suggestive evidence of self-recognition in bottlenose dolphins. Diverse species such as elephants (Povinelli, 1989), and African grey parrots (Pepperberg, 1993) have demonstrated mirror guided behaviour in accomplishing tasks.

Evidence for conceptual quantitative abilities has been reported in the African grey parrot (Pepperberg, 1987) and the chimpanzee (Boysen and Berntson, 1989).

There is clearly much more complexity and plasticity found in the communication systems of other species that previously thought. More research is needed with more diverse species to more adequately access the extent to which referential communication, quantitative abilities and complex information processing has developed throughout the biological world.

\section{References}

Boysen, S.T. and Berntson, G,G. (1989). Numerical Competence in a chimpanzee (Pan troglodytes). Journal of Comparative Psychology, 103, 23-30.

Fouts, R.S. (1973). Acquisition and testing of gestural signs in four young chimpanzees. Science, 180: 978-980.

Gardner, R.A. and Gardner, B.T. (1969). Teaching sign language to a chimpanzee. Science, 1965: 664-672.

Marion, L.A., Reiss, D. and Gallup, Jr. G.G. (1993). A test of self recognition in dolphins: implications for comparative investigations of highly dissimilar species. In: Selfawareness in Humans and Nonhumans. S. Parker, R. Mitchell, and M. Boccia (eds.) Cambridge University Press.

Marino, L.A., (1993) SETI begins at home: Can we define and measure intelligence among species $\mathrm{n}$ this planet? 1993 Bioastronomy Symposium, University of California, Santa Cruz. 
Owings, D.H. and Lager, D.W. (1980). Chatter vocalizations of California ground squirrels: Predator and social role - specificity. Zeitschrift fur Tierpsychologie, 54, 163-184.

Pepperberg, I.M. (1981) Functional vocalizations by an African abilities in the African parrot: labelling cardinal sets. Ethology, 75, 37-61.

Povinelli, D.J. (1987) Monkeys, apes, mirrors and minds: The evolution of self-awareness on primates. Human Evolution, 2, 493-509.

Povinelli, D.J. (1989). Failure to find self-recognition in Asian Elephants (Elephas maximus) in contrast to their use in mirror cues to discover hidden food. Journal of Comparative Psychology, , 103, 122-131.

Reiss, D. (1988). Can we communicate with other species on this planet: pragmatics of communication between humanoid and nonhumanoid species. In Biostronomy - The Next Steps (G. Marx, ed.) Kluwer Academic Pubs. 253-264.

Reiss, D, and McCowan, B. (1993) Spontaneous vocal mimicry and production by Bottlenose Dolphins (Tursiops truncatus). evidence for vocal learning, Journal of Comparative Psychology. 107, 301-312.

Reiss, D. (1993a) Studying the communication signals of bottlenose dolphins (Tursiops truncatus). 1993 Bioastronomy Symposium, University of California, Santa Cruz.

Richards, D.G. Wolz, J.P. and Herman, L.M. Vocal mimicry of computer generated sounds and vocal labelling of objects by a bottlenose dolphin. Journal of Comparative Psychology, 98, 10-28.

Savage-Rambaugh, E.S. (1986). Ape language: From conditioned response to symbol. New York: Columbia University Press

Schusterman, R.J. and Krieger, K. (1984) California sea lions are capable of semantic comprehension. The Psychological Record, 34, 3-23.

Seyfarth, R.M., Cheney, D.L. and Marier, P. (1980). Vervet monket alarm calls; semantic communication in a free-ranging primate. Animal Behavior, 28, 1070-1094.

\section{SETI: J. Tarter}

During the past triennium there have been nine SETI observational programs active around the world. Some of them are literally backyard operations, and others are major undertakings utilizing many large telescope facilities. Most, but not all, are conducted at radio frequencies and involve the US, Argentina, and Australia. The following tables summarize the characteristics of these nine programs, which are listed in order of the observational start date.

TABLE 1- OSURO; Ohio State University Radio Observatory

PI's: Prof. Robert Dixon and Prof. John Kraus

ANTENNA: Kraus design / $52 \mathrm{~m}$ parabolic equivalent

FREQUENCY COVERAGE: $1400-1700 \mathrm{MHz}$

POLARIZATION: single linear

FREQUENCY RESOLUTION: $100 \mathrm{kHz}$

SPECTROMETER: 3000 channel filter bank

SENSITIVITIY: $1.5 \times 10^{-21} \mathrm{~W} / \mathrm{m}^{2}$

SEARCH STRATEGY: Sky Survey at sidereal rate. Data passing threshold stored for later analysis. Tracking mode is automatically triggered to follow an "interesting" signal until it sets.

TABLE 2 - Small SETI Observatory 
Owings, D.H. and Lager, D.W. (1980). Chatter vocalizations of California ground squirrels: Predator and social role - specificity. Zeitschrift fur Tierpsychologie, 54, 163-184.

Pepperberg, I.M. (1981) Functional vocalizations by an African abilities in the African parrot: labelling cardinal sets. Ethology, 75, 37-61.

Povinelli, D.J. (1987) Monkeys, apes, mirrors and minds: The evolution of self-awareness on primates. Human Evolution, 2, 493-509.

Povinelli, D.J. (1989). Failure to find self-recognition in Asian Elephants (Elephas maximus) in contrast to their use in mirror cues to discover hidden food. Journal of Comparative Psychology, , 103, 122-131.

Reiss, D. (1988). Can we communicate with other species on this planet: pragmatics of communication between humanoid and nonhumanoid species. In Biostronomy - The Next Steps (G. Marx, ed.) Kluwer Academic Pubs. 253-264.

Reiss, D, and McCowan, B. (1993) Spontaneous vocal mimicry and production by Bottlenose Dolphins (Tursiops truncatus). evidence for vocal learning, Journal of Comparative Psychology. 107, 301-312.

Reiss, D. (1993a) Studying the communication signals of bottlenose dolphins (Tursiops truncatus). 1993 Bioastronomy Symposium, University of California, Santa Cruz.

Richards, D.G. Wolz, J.P. and Herman, L.M. Vocal mimicry of computer generated sounds and vocal labelling of objects by a bottlenose dolphin. Journal of Comparative Psychology, 98, 10-28.

Savage-Rambaugh, E.S. (1986). Ape language: From conditioned response to symbol. New York: Columbia University Press

Schusterman, R.J. and Krieger, K. (1984) California sea lions are capable of semantic comprehension. The Psychological Record, 34, 3-23.

Seyfarth, R.M., Cheney, D.L. and Marier, P. (1980). Vervet monket alarm calls; semantic communication in a free-ranging primate. Animal Behavior, 28, 1070-1094.

\section{SETI: J. Tarter}

During the past triennium there have been nine SETI observational programs active around the world. Some of them are literally backyard operations, and others are major undertakings utilizing many large telescope facilities. Most, but not all, are conducted at radio frequencies and involve the US, Argentina, and Australia. The following tables summarize the characteristics of these nine programs, which are listed in order of the observational start date.

TABLE 1- OSURO; Ohio State University Radio Observatory

PI's: Prof. Robert Dixon and Prof. John Kraus

ANTENNA: Kraus design / $52 \mathrm{~m}$ parabolic equivalent

FREQUENCY COVERAGE: $1400-1700 \mathrm{MHz}$

POLARIZATION: single linear

FREQUENCY RESOLUTION: $100 \mathrm{kHz}$

SPECTROMETER: 3000 channel filter bank

SENSITIVITIY: $1.5 \times 10^{-21} \mathrm{~W} / \mathrm{m}^{2}$

SEARCH STRATEGY: Sky Survey at sidereal rate. Data passing threshold stored for later analysis. Tracking mode is automatically triggered to follow an "interesting" signal until it sets.

TABLE 2 - Small SETI Observatory 
PI's: Robert Grey

LOCATION: Chicago, Illinois

ANTENNA: $4 \mathrm{~m}$ satellite dish

FREQUENCY COVERAGE:

POLARIZATION: single linear

FREQUENCY RESOLUTION: variable from $100 \mathrm{~Hz}$ to $1 \mathrm{~Hz}$

SENSITIVITIY: $10^{-21} \mathrm{~W} / \mathrm{m}^{2}$

SEARCH STRATEGY: Concentration on area of sky from whence the OSURO 1977 "wow" signal appeared to originate, also searches of M31 and M33.

TABLE 3 - META Mega-Channel ExtraTerrestrial Assay

PI's: Prof. Paul Horowitz, Harvard University

LOCATION: Oak Ridge Observatory in Harvard, Massachusetts

ANTENNA: $26 \mathrm{~m} 200 \mathrm{kHz}$

POLARIZATION: sequential, dual circular

FREQUENCY RESOLUTION: $0.05 \mathrm{~Hz}$

SPECTROMETER: 8,388,608 channel FFT spectrometer with continuous self-test and mappable, redundant processors

SENSITIVITIY: $7 \times 10^{-24} \mathrm{~W} / \mathrm{m}^{2}$

SEARCH STRATEGY:Sky Survey at sidereal rate over $68 \%$ of sky (-30 to +60 degrees declination), moving $1 / 2$ degree per day. Reobservation within minutes for signals exceeding 20 sigma. Magic frequencies sequentially observed with respect toHeliocentric, Galactocentric and $3 \mathrm{~K}$ Blackbody Background frames of rest.

TABLE 4 - META II

PI's: Prof. F. Raul Colomb

LOCATION: Instituto Argentino de Radioastronomia, Buenos Aires, Argentina

ANTENNA: $30 \mathrm{~m}$ fully steerable parabola

FREQUENCY COVERAGE: $1420 \mathrm{MHz}+/-200 \mathrm{kHz}$

POLARIZATION: sequential, dual circular

FREQUENCY RESOLUTION: $0.05 \mathrm{~Hz}$

SPECTROMETER: META clone, $8,388,608$ channel FFT spectrometer

SENSITIVITIY: $4 \times 10^{-24} \mathrm{~W} / \mathrm{m}^{2}$

SEARCH STRATEGY: Half of observatory time spent on sky survey of southern sky for magic frequency signal in same manner as META in the North 


\section{TABLE 5 - TMTS Ten Micron Targeted Search}

PI's: Prof. Albert Betz, University of Colorado, Boulder

LOCATION: Mount Wilson Observatory, Pasadena, CA

ANTENNA: $1.7 \mathrm{~m}$ (one of two)

FREQUENCY COVERAGE: 10 microns (30 THz $+/-60 \mathrm{~km} / \mathrm{s}$ )

POLARIZATION: single linear

FREQUENCY RESOLUTION: 2.4 and $5 \mathrm{MHz}$

SPECTROMETER: Heterodyned, acousto-optical spectrometers with either 1024 or 2048 channels SENSITIVITIY: $9 \times 10^{-18} \mathrm{~W} / \mathrm{m}^{2}$

SEARCH STRATEGY: Targeted Search of 200 nearby, solar-type stars, looking for intentional $\mathrm{CO} 2$ laser signals

TABLE 6 - Search for Interstellar Communication Channel

PI's: Prof. David Blair, University of Westem Australia

LOCATION: Parkes Observatory, NSW Australia

ANTENNA: $64 \mathrm{~m}$

FREQUENCY COVERAGE: $4462.3 \mathrm{MHz}$ (pi x HI)

POLARIZATION: two linear polirizations with three different frames of reference spread between them FREQUENCY RESOLUTION: $100 \mathrm{~Hz}$

SPECTROMETER: $2 \times 512$ channel correlator and HP3582A spectrum analyser

SENSITIVITIY: $2 \times 10^{-24} \mathrm{~W} / \mathrm{m}^{2}$

SEARCH STRATEGY: Targeted search of -100 southern, nearby, solar-type stars

TABLE 7 - SERENDIP III: Search for Extraterrestrial Radio Emission from Nearby Developed Intelligent Populations

PI's: Prof. Stuart Bowyer, University of California Berkeley

LOCATION: Arecibo Observatory, Puerto Rico

ANTENNA: $305 \mathrm{~m}$ spherical dish

FREQUENCY COVERAGE: $423-435 \mathrm{MHz}$

POLARIZATION: single linear

FREQUENCY RESOLUTION: 0.6 HzSPECTROMETER: 4.2 million channel FFT spectometer SENSITIVITIY: $6 \times 10^{-25} \mathrm{~W} / \mathrm{m}^{2}$ 
SEARCH STRATEGY: Commensal search, non-directed sky survey at twice negative sidereal rate

TABLE 8A - HRMS SS: High Resolution Microwave Survey Sky Survey

PI's: Project Scientist for SS is Dr. Sam Gulkis, JPL

LOCATION: DSS13 at Goldstone DSN Complex in Mohave Desert

ANTENNA: $34 \mathrm{~m}$ beam-waveguide

FREQUENCY COVERAGE: X-band $+/-40 \mathrm{MHz}$

POLARIZATION: sequential, dual circular

FREQUENCY RESOLUTION: $20 \mathrm{~Hz}$

SPECTROMETER: 2 million channel FFT spectrometer

SENSITIVITTY: $3 \times 10^{-23} \mathrm{~W} / \mathrm{m}^{2}$

SEARCH STRATEGY: Sky Survey of northern hemisphere at 0.2 degree per second, using "racetrack" scans of invividual skyframes. Largest events above threshold along a scan line and co-located on adjacent scan lines are followed up with targeted observations.

TABLE 8B - HRMS TS: High Resolution Microwave Survey Targeted Search

PI's: The Project Scientist for HRMS is Dr. Jill Tarter, Ames Research Center

LOCATION: Arecibo Observatory, Puerto Rico

ANTENNA: $213 \mathrm{~m}$ spherical dish

FREQUENCY COVERAGE: $300 \mathrm{MHz}$ in range 1300 to $2300 \mathrm{MHz}$

POLARIZATION: dual circular

FREQUENCY RESOLUTION: 1,7 and $28 \mathrm{~Hz}$ simulataneously

SPECTROMETER: 14 million channel FFT spectrometer at $1 \mathrm{~Hz}, 2$ million channel spectrometer at $7 \mathrm{~Hz}$ and .5 million channel spectrometer at $28 \mathrm{~Hz}$

SENSITIVITIY: $3 \times 10^{-27} \mathrm{~W} / \mathrm{m}^{2}$

SEARCH STRATEGY: Targeted Search of an initial list of 25 solar-type stars passing directly overhead at Arecibo searching for narrowband pulses and $\mathrm{CW}$ signals that may be drifting slowly in frequency.

TABLE 9 - Columbus Optical SETI Observatory

PI': Dr. Stuart Kingsley

LOCATION: Columbus, Ohio (home address)

ANTENNA: 10" Meade LX200 Schmidt-Cassegrain

FREQUENCY COVERAGE: Optical and near IR

POLARIZATION: - 
FREQUENCY RESOLUTION: -

SPECTROMETER: -

SENSITIVITIY: -

SEARCH STRATEGY: This facility is so new that it has not yet begun a formal search program. 